\title{
MULTIPLE SCATTERING POLARIZATION OF SUBSTELLAR-MASS OBJECTS: T- DWARFS
}

\author{
Sujan Sengupta \\ Indian Institute of Astrophysics, Koramangala 2nd Block, Bangalore 560 034, India; \\ sujan@iiap.res.in \\ and \\ Mark S. Marley \\ NASA Ames Research Center, MS-245-3, Moffett Field, CA 94035, U.S.A.; \\ Mark.S.Marley@NASA.gov
}

\begin{abstract}
While there have been multiple observational programs aimed at detecting linear polarization of optical radiation emitted by ultracool dwarfs, there has been comparatively less rigorous theoretical analysis of the problem. The general expectation has been that the atmospheres of those substellar-mass objects with condensate clouds would give rise to linear polarization due to scattering. Because of rotation-induced non-sphericity, there is expected to be incomplete cancellation of disk-integrated net polarization and thus a finite polarization. For cloudless objects, however, only molecular Rayleigh scattering will contribute to any net polarization and this limit has not been well studied. Hence in this paper we present a detailed multiple scattering analysis of the polarization expected from those T-dwarfs whose spectra show absence of condensates. For this, we develop and solve the full radiative transfer equations for linearly polarized radiation. Only atomic and molecular Rayleigh scattering are considered to be the source of polarization. We compute the local polarization at different angular directions in a plane-parallel atmospheres calculated for the range of effective temperatures of $\mathrm{T}$ dwarfs and then average over the whole surface of the object. The effects of gravity and limb darkening as well as rotation induced non-sphericity are included. It is found that the amount of polarization decreases with the increase in effective temperature. It is also found that significant polarization at any local point in the atmosphere arises only in the optical (B-band). However, the disk integrated polarization-even in the B-band-is negligible. Hence we conclude that, unlike the case for cloudy L dwarfs, polarization of cloudless T-dwarfs by atomic and molecular scattering may not be detectable. In the future we will extend this work to cloudy L- and T-dwarf atmospheres.
\end{abstract}

Subject headings: stars: low mass, brown dwarfs - polarization, extinction — scattering — stars : atmospheres 


\section{INTRODUCTION}

Brown dwarfs have masses sufficient to ignite deuterium burning but insufficient to enter into the hydrogen burning main sequence. Instead, they radiate away the gravitational potential energy produced during their formation and contraction. As an individual brown dwarf cools it progressively passes through spectral types ranging from late $\mathrm{M}$ through the $\mathrm{L}$ and $\mathrm{T}$ sequences. Helpful reviews of brown dwarf science may be found in Chabrier \& Baraffe (2000), Burrows et al. (2001), Kirkpatrick (2005), and Marley \& Leggett (2008).

While condensates such as iron, forsterite $\left(\mathrm{Mg}_{2} \mathrm{SiO}_{4}\right)$, and enstatite $\left(\mathrm{MgSiO}_{3}\right)$ are favored to be formed in the atmosphere of relatively warm L dwarfs (Lunine. Hubbard \& Marley 1986; Tsuji et al. 1996; Lodders, K. 1999; Burrows, Marley \& Sharp 2000; Allard et al. 2001), in the atmosphere of mid-type and later $\mathrm{T}$ dwarfs, grains condense well bellow the photosphere and are not an important opacity source (Ackerman \& Marlev 2001; Tsuii 2002; Chabrier \& Baraffe 2000; Burrows et al. 2001; Kirkpatrick 2005; Marley \& Leggett 2008). Synthetic spectra of model atmospheres lacking condensates fit well with the observed spectra of field $\mathrm{T}$ dwarfs later than about type T4 (with effective temperature $T_{\text {eff }} \sim 1200 \mathrm{~K}$; Stephens et al. (2009)) suggesting the absence of condensates in the visible atmosphere of these objects. At the same time, inclusion of condensate cloud of various species explains the spectra of L dwarfs (e.g., Cushing et al. 2008).

Sengupta \& Krishan (2001) first predicted that scattering of light by the condensates in the photopshere of L dwarfs would give rise to a significant amount of linear polarization. This polarization would of course cancel out when integrated over a spherical surface of the star. However, analysis of high resolution spectra of $\mathrm{L}$ dwarfs confirms that these objects are rapid rotator with the projected rotational velocity ranging from as high as 90 to $10 \mathrm{kms}^{-1}$ (Basri et al. 2000; Mohanty \& Basri 2003; Zapatero Osorio et al. 2006; Reiners \& Basri 2008; Bailer-Jones 2004). A rapid rotation around its axis induces departure from sphericity in the shape of the object. As a consequence, the net polarization integrated over the disk does not completely cancel out. Subsequently, linear polarization of several L dwarfs has been detected by various group of astronomers \begin{tabular}{|l|l|l|l|} 
(Menard et al. 2002; Zapatero Osorio et al. 2005; Goldman et al. 2009; Tata et al. 2009). The
\end{tabular} observation were analyzed and explained by Sengupta (2003); Sengupta \& Kwok (2005) who assumed a single scattering atmosphere wherein the effect of atoms and molecules was neglected. These authors suggested that scattering by atoms and molecules would give rise to negligible amount of polarization and hence polarization of $\mathrm{T}$ dwarfs in the optical should be inconsequential. However their single scattering approach left room for some uncertainty because they considered a purely scattering medium while a proper approach would incorporate both absorption and multiple scattering.

In this paper we present the results of our detailed investigation on the polarization of T-dwarfs caused by multiple scattering of atoms and molecules in cloud-less atmospheres. In the next section we discuss the atmospheric models. In section 3, we present in detail the equations of transfer of polarized radiation and in section 4 we present the numerical methods of solving the transfer 
equation of polarized radiation in a plane-parallel medium. The local polarization is integrated over the rotationally distorted surface. We briefly describe the method adopted to calculate the rotational oblateness and the methods of integrating the polarization over a rotationally distorted stellar disk in section 5 . We discuss the results in section 6 followed by our conclusion in the last section.

\section{ATMOSPHERIC MODELS OF T-DWARFS}

The known field $\mathrm{T}$ dwarfs have effective temperatures in the range $1400>T_{\text {eff }}>550 \mathrm{~K}$. Clouds influence the observed spectra of the warmest dwarfs, but cloudless models generally reproduce the spectra of most $\mathrm{T}$ dwarfs with $T_{\text {eff }}<1200 \mathrm{~K}$, although this temperature is likely gravity dependent (Stephens et al. 2009). For the study reported here we considered only cloudless models appropriate for spectral types later than about T3 (Stephens et al. 2009).

For the polarization study we used four radiative-convective equilibrium models which give the run of temperature as a function of pressure through the atmosphere from the collection employed by Saumon \& Marley (2008) in their study of brown dwarf evolution. The selected models include no cloud opacity, although condensates are included in the chemical equilibrium calculation (Freedman. Marlev and Lodders 2008). A more detailed summary of the model atmospheres is presented in Stephens et al. (2009). The models shown here all have $\log g=5$ and solar metallicity. The effect of variation in these parameters will be explored in a future study. Here we choose models with $T_{\text {eff }}=1400,1200,1000$, and $800 \mathrm{~K}$. As noted above, the first is somewhat warm for a typical cloudless field $\mathrm{T}$ dwarf but we include it as a comparison point for future studies of cloudy L dwarfs with this $T_{\text {eff }}$.

The four model temperature-pressure profiles described above were then used as inputs for the polarization radiative transfer model. The same atmospheric opacities employed in the calculation of the radiative-convective equilibrium models (Freedman, Marley and Lodders 2008) were used in both calculations. We note that in the calculation of these temperature profiles we computed fluxes in 180 separate spectral bins. Within each bin we computed the radiative transfer 8 times, once for each of eight k-coefficient gauss points (Marlev et al. 2002). The details of the underlying opacity calculation are presented in Freedman, Marley and Lodders (2008). For the polarization study we followed this same procedure and computed monochromatic intensities at centers of the same 180 flux bins using the same opacities. Final computed quantities are the gauss weighted sum of the eight independent radiative transfer calculations at each of the 180 wavelength points. We note that while the resulting spectrum has somewhat low spectral resolution, polarization changes slowly with wavelength and this resolution is adequate for our purposes. 


\section{EQUATIONS OF TRANSFER OF POLARIZED RADIATION}

\subsection{Representation of polarized radiation by Stokes parameters}

In order to formulate the equations of transfer in a gaseous medium the most convenient representation of polarized radiation is by a set of four parameters called Stokes parameters. Chandrasekhar (1960) first introduced the Stokes parameters in the equation of radiative transfer with a slight modification of Stoke's representation. In an elliptically polarized beam, the vibrations of the electric and the magnetic vectors in the plane transverse to the direction of propagation are such that the ratio of the amplitudes and difference in phases of the components in any two directions at right angles to each other are absolute constants. A regular vibration of this character can be represented by

$$
\xi_{l}=\xi_{l}^{(0)} \sin \left(\omega t-\epsilon_{l}\right), \xi_{r}=\xi_{r}^{(0)} \sin \left(\omega t-\epsilon_{r}\right)
$$

where $\xi_{l}$ and $\xi_{r}$ are the components of the vibration along two directions $l$ and $r$ at right angles to

each other, $\omega$ the circular frequency of the vibration, and $\xi_{l}^{(0)}, \xi_{r}^{(0)}, \epsilon_{l}$ and $\epsilon_{r}$ are constants. If the principal axes of the ellipse described by $\left(\xi_{l}, \xi_{r}\right)$ are in directions making angles $\chi$ and $\chi+\frac{1}{2} \pi$ to the direction $l$, the equations representing the vibration take the simplified forms :

$$
\xi_{\chi}=\xi^{(0)} \cos \beta \sin \omega t, \xi_{\chi+\frac{1}{2} \pi}=\xi^{(0)} \sin \beta \cos \omega t
$$

where $\beta$ denotes an angle whose tangent is the ratio of the axes of the ellipse traced by the end point of the electric (or magnetic) vector, and the numerical values of it lies between 0 and $\frac{1}{2} \pi$. The sign of $\beta$ is positive or negative according as the polarization is right-handed or left-handed.

In equation (2), $\xi^{(0)}$ denotes a quantity proportional to the mean amplitude of the electric vector and whose square is equal to the intensity of the beam:

$$
I=\left[\xi^{(0)}\right]^{2}=\left[\xi_{l}^{(0)}\right]^{2}+\left[\xi_{r}^{(0)}\right]^{2}=I_{l}+I_{r}
$$

Following the representation given in equation (2) one obtains for the vibrations in the $l$ and $r$ directions

$$
\xi_{l}=\xi^{(0)}(\cos \beta \cos \chi \sin \omega t-\sin \beta \sin \chi \cos \omega t)
$$

and

$$
\xi_{r}=\xi^{(0)}(\cos \beta \sin \chi \sin \omega t-\sin \beta \cos \chi \cos \omega t) .
$$

The intensities $I_{l}$ and $I_{r}$ in the directions $l$ and $r$ can be written as

$$
I_{l}=\left[\xi_{l}^{(0)}\right]^{2}=I\left(\cos ^{2} \beta \cos ^{2} \chi+\sin ^{2} \beta \sin ^{2} \chi\right)
$$


and

$$
I_{r}=\left[\xi_{r}^{(0)}\right]^{2}=I\left(\cos ^{2} \beta \sin ^{2} \chi+\sin ^{2} \beta \cos ^{2} \chi\right)
$$

From the previous equations it follows that if the regular vibrations representing an elliptically polarized beam can be expressed in the form given in equation (11), we can write the relations

$$
\begin{gathered}
I=\left[\xi_{l}^{(0)}\right]^{2}+\left[\xi_{r}^{(0)}\right]^{2}=I_{l}+I_{r} \\
Q=\left[\xi_{l}^{(0)}\right]^{2}-\left[\xi_{r}^{(0)}\right]^{2}=I \cos 2 \beta \cos 2 \chi=I_{l}-I_{r} \\
U=2 \xi_{l}^{(0)} \xi_{r}^{(0)} \cos \left(\epsilon_{l}-\epsilon_{r}\right)=I \cos 2 \beta \sin 2 \chi=\left(I_{l}-I_{r}\right) \tan 2 \chi
\end{gathered}
$$

and

$$
V=2 \xi_{l}^{(0)} \xi_{r}^{(0)} \sin \left(\epsilon_{l}-\epsilon_{r}\right)=I \sin 2 \beta=\left(I_{l}-I_{r}\right) \tan 2 \beta \sec 2 \chi .
$$

These are the Stokes parameters representing an elliptically polarized beam. It follows from equation (8) to equation (11) that

$$
I^{2}=Q^{2}+U^{2}+V^{2}
$$

Further,

$$
\tan 2 \chi=\frac{U}{Q}
$$

and

$$
\sin 2 \beta=\frac{V}{\sqrt{Q^{2}+U^{2}+V^{2}}}
$$

which give the plane of polarization and the ellipticity respectively.

The nature of an arbitrary polarized light is completely determined by the intensities in two directions at right angles to each other (or equivalently, the total intensity $I$ and $Q=I_{l}-I_{r}$ ) and the parameters $U$ and $V$. The intensities $I, Q, U$ and $V$ are the general Stokes parameters representing light.

\subsection{Rayleigh scattering}

In order to incorporate Rayleigh's scattering into the radiative transfer equation, Chandrasekhar (1960) modified it which can be stated as the vibrations representing the light scattered in a direction making an angle $\Theta$ with the direction of incidence is

$$
\xi_{\|}^{(0)}=\left(\frac{3}{2} \sigma\right)^{1 / 2} \xi_{\|}^{(0)} \cos \Theta \sin \left(\omega t-\epsilon_{1}\right)
$$


and

$$
\xi_{\perp}^{(0)}=\left(\frac{3}{2} \sigma\right)^{1 / 2} \xi_{\perp}^{(0)} \sin \left(\omega t-\epsilon_{2}\right)
$$

where the phase $\left(\epsilon_{1}, \epsilon_{2}\right)$ and the amplitude $\left(\xi_{\|}^{(0)}, \xi_{\perp}^{(0)}\right)$, relations in the incident beam are maintained, unaltered, in the scattered beam. Here $\|$ and $\perp$ refer to directions in the transverse planes (of the incident and scattered light) parallel and perpendicular respectively to the plane of scattering. Accordingly, the parameters representing the scattered light are proportional to

$$
\begin{gathered}
\frac{3}{2} \sigma\left[\overline{\xi_{\|}^{(0)}}\right]^{2} \cos ^{2} \Theta=\frac{3}{2} \sigma I_{\|} \cos ^{2} \Theta, \\
\frac{3}{2} \sigma\left[\overline{\xi_{\perp}^{(0)}}\right]^{2}=\frac{3}{2} \sigma I_{\perp}, \\
\frac{3}{2} \sigma\left[\overline{2 \xi_{\|}^{(0)} \xi_{\perp}^{(0)} \cos \left(\epsilon_{1}-\epsilon_{2}\right)}\right] \cos \Theta=\frac{3}{2} \sigma U \cos \Theta
\end{gathered}
$$

and

$$
\frac{3}{2} \sigma\left[\overline{2 \xi_{\|}^{(0)} \xi_{\perp}^{(0)} \sin \left(\epsilon_{1}-\epsilon_{2}\right)}\right] \cos \Theta=\frac{3}{2} \sigma V \cos \Theta
$$

Therefore, denoting the incident light by the vector

$$
\mathbf{I}=\left(I_{\|}, I_{\perp}, U, V\right)
$$

we can express the scattering intensity in the direction $\Theta$ by

$$
\left(\sigma \frac{d \omega^{\prime}}{4 \pi}\right) \mathbf{R I} d \omega
$$

where

$$
\mathbf{R}=\frac{3}{2}\left(\begin{array}{cccc}
\cos ^{2} \Theta & 0 & 0 & 0 \\
0 & 1 & 0 & 0 \\
0 & 0 & \cos \Theta & 0 \\
0 & 0 & 0 & \cos \Theta
\end{array}\right)
$$

$\mathbf{R}$ is called the phase matrix for Rayleigh scattering.

\subsection{The explicit form of the phase matrix for Rayleigh scattering}

In the formulation of the equation of radiative transfer, the radiation field at each point is characterized by the four intensities $I_{l}(\theta, \phi), I_{r}(\theta, \phi), U(\theta, \phi)$ and $V(\theta, \phi)$ where $\theta$ and $\phi$ are the polar angles referred to an appropriately chosen coordinate system through the point under 
consideration and $l$ and $r$ refer to the directions in the meridian plane and at right angles to it respectively. Therefore one writes

$$
\mathbf{I}(\theta, \phi)=\left[I_{l}(\theta, \phi), I_{r}(\theta, \phi), U(\theta, \phi), V(\theta, \phi)\right] .
$$

The explicit form of the phase function for Rayleigh scattering in terms of $\theta$ and $\phi$ which is used in the transfer equation and which describes the angular distribution of the radiation field is given by (Chandrasekhar 1960):

$$
\begin{aligned}
P\left(\mu, \phi ; \mu^{\prime}, \phi^{\prime}\right)= & Q\left[P^{(0)}\left(\mu, \mu^{\prime}\right)+\left(1-\mu^{2}\right)^{1 / 2}\left(1-\mu^{\prime 2}\right)^{1 / 2} P^{(1)}\left(\mu, \phi ; \mu^{\prime}, \phi^{\prime}\right)\right. \\
& \left.+P^{(2)}\left(\mu, \phi ; \mu^{\prime}, \phi^{\prime}\right)\right]
\end{aligned}
$$

where

$$
\begin{aligned}
& P^{(0)}\left(\mu, \mu^{\prime}\right)=\frac{3}{4}\left(\begin{array}{cccc}
2\left(1-\mu^{2}\right)\left(1-\mu^{\prime 2}\right)+\mu^{2} \mu^{\prime 2} & \mu^{2} & 0 & 0 \\
\mu^{\prime 2} & 1 & 0 & 0 \\
0 & 0 & 0 & 0 \\
0 & 0 & 0 & \mu \mu^{\prime}
\end{array}\right) \\
& P^{(1)}\left(\mu, \phi ; \mu^{\prime}, \phi^{\prime}\right)=\frac{3}{4}\left(\begin{array}{cccc}
4 \mu \mu^{\prime} \cos \left(\phi^{\prime}-\phi\right) & 0 & 2 \mu \sin \left(\phi^{\prime}-\phi\right) & 0 \\
0 & 0 & 0 & 0 \\
-2 \mu^{\prime} \sin \left(\phi^{\prime}-\phi\right) & 0 & \cos \left(\phi^{\prime}-\phi\right) & 0 \\
0 & 0 & 0 & \cos \left(\phi^{\prime}-\phi\right)
\end{array}\right) \\
& P^{(2)}\left(\mu, \phi ; \mu^{\prime}, \phi^{\prime}\right)=\frac{3}{4}\left(\begin{array}{cccc}
\mu^{2} \mu^{\prime 2} \cos 2\left(\phi^{\prime}-\phi\right) & -\mu^{2} \cos 2\left(\phi^{\prime}-\phi\right) & \mu^{2} \mu^{\prime} \sin 2\left(\phi^{\prime}-\phi\right) & 0 \\
-\mu^{\prime 2} \cos 2\left(\phi^{\prime}-\phi\right) & \cos 2\left(\phi^{\prime}-\phi\right) & -\mu^{\prime} \sin 2\left(\phi^{\prime}-\phi\right) & 0 \\
-\mu \mu^{\prime 2} \sin 2\left(\phi^{\prime}-\phi\right) & \mu \sin 2\left(\phi^{\prime}-\phi\right) & \mu \mu^{\prime} \cos 2\left(\phi^{\prime}-\phi\right) & 0 \\
0 & 0 & 0 & 0
\end{array}\right)
\end{aligned}
$$

and

$$
Q=\left(\begin{array}{llll}
1 & 0 & 0 & 0 \\
0 & 1 & 0 & 0 \\
0 & 0 & 2 & 0 \\
0 & 0 & 0 & 2
\end{array}\right)
$$

Here $\mu=\cos \theta, \mu^{\prime}=\cos \theta^{\prime}, \theta$ and $\theta^{\prime}$ denote the direction of the photon before and after scattering. Similarly for $\phi$ and $\phi^{\prime}$.

In case of the axial symmetry of the radiation field, it clearly requires that the plane of polarization be along the meridian plane (or, at right angle to it). Consequently, $U=V=0$ and the two intensities $I_{l}$ and $I_{r}$ are sufficient to characterize the radiation field.

With this consideration the phase matrix used to describe the angular distribution of photons that undergo Rayleigh scattering can be written as

$$
\mathbf{P}\left(\mu, \mu^{\prime}\right)=\frac{3}{4}\left(\begin{array}{cc}
2\left(1-\mu^{2}\right)\left(1-\mu^{\prime 2}\right)+\mu^{2} \mu^{\prime 2} & \mu^{2} \\
\mu^{\prime 2} & 1
\end{array}\right)
$$




\subsection{Equations for the transfer of polarized radiation in plane- parallel geometry}

In the present work, plane parallel atmosphere has been considered. The transfer equation governing the intensities $I_{l}$ and $I_{r}$ in rest frame with the medium stratified into plane parallel can be written as

$$
\mu \frac{\partial}{\partial \tau}\left(\begin{array}{c}
I_{l}(\mu) \\
I_{r}(\mu)
\end{array}\right)=\left(\begin{array}{c}
I_{l}(\mu) \\
I_{r}(\mu)
\end{array}\right)-\frac{\omega_{0}}{2} \int_{-1}^{1} \mathbf{P}\left(\mu, \mu^{\prime}\right)\left(\begin{array}{c}
I_{l}(\mu) \\
I_{r}(\mu)
\end{array}\right) d \mu^{\prime}-\left(1-\omega_{0}\right) \mathbf{b}(\mathbf{z})
$$

where $\mu=\cos \theta(\mu \in[0,1])$ and $\theta$ is the angle between the Stokes specific intensity vector and the axis of symmetry $z ; \mathbf{b}(\mathbf{z})$ is the internal radiation source, $\omega_{0}$ is the albedo for single scattering and $\tau$ is the optical depth.

In case of spherical geometry the equation of transfer for polarized radiation can be written as

$$
\begin{aligned}
& \mu \frac{\partial}{\partial r}\left(\begin{array}{c}
I_{l}(\mu) \\
I_{r}(\mu)
\end{array}\right)+\frac{1-\mu^{2}}{r} \frac{\partial}{\partial \mu}\left(\begin{array}{c}
I_{l}(x, \mu, r) \\
I_{r}(x, \mu, r)
\end{array}\right)= \\
& -\kappa \rho\left\{\left(\begin{array}{c}
I_{l}(\mu) \\
I_{r}(\mu)
\end{array}\right)-\frac{\omega_{0}}{2} \int_{-1}^{1} \mathbf{P}\left(\mu, \mu^{\prime}\right)\left(\begin{array}{c}
I_{l}(\mu) \\
I_{r}(\mu)
\end{array}\right) d \mu^{\prime}-\left(1-\omega_{0}\right) \mathbf{b}(\mathbf{z})\right\}
\end{aligned}
$$

where $\kappa$ is the mass absorption co-efficient for radiation of frequency $\nu$ and $\rho$ is the density of the material such that between the points $s$ and $s^{\prime}$,

$$
\tau\left(s, s^{\prime}\right)=\int_{s^{\prime}}^{s} \kappa \rho d s
$$

For an object having radius as small as that of Jupiter with geometrically thin atmosphere, the second term in the left hand side of the above equation which incorporates the curvature effect becomes negligible. Therefore, for solar type of stars and substellar-mass objects, the plane-parallel stratification is sufficient.

The degree of linear polarization $\mathrm{p}$ is given by

$$
p(\mu)=\frac{I_{l}(\mu)-I_{r}(\mu)}{I_{l}(\mu)+I_{r}(\mu)}
$$

\section{NUMERICAL METHODS}

The work of Redheffer (1962) and Preisendorfer (1965) on the interaction principle have been formalized by Grant \& Hunt (1969), Grant \& Hunt (1969a) with the introduction of the internal sources which is crucial for the stellar atmospheres. Following this work, Grant \& Peraiah (1972) and Peraiah \& Grant (1973) developed a method to obtain direct solution of the transfer equations. This method is called the discrete space theory of Radiative Transfer. In the present 
work the method due to Peraiah \& Grant (1973) has been employed to solve the radiative transfer equations in their vector form for linear polarization.

In this method, the entire medium is divided into $\mathrm{N}$ number of shells and it is assumed that the specific intensities $I_{n}^{+}$and $I_{n+1}^{-}$are incident at the boundaries $n$ and $n+1$ respectively of the shell with optical thickness $\tau$. The symbols with signs + and - represent specific intensities of the rays travelling in opposite directions. If $\mu$ represents the cosine of the angle made by a ray with the normal to the plane parallel layers in the direction in which the geometrical depth decreases. That is,

$$
I_{n}^{+}\left[I_{n}(\mu): 0<\mu \leq 1\right]
$$

and

$$
I_{n}^{-}\left[I_{n}(-\mu): 0<\mu \leq 1\right],
$$

$I_{n}^{+}$represents the specific intensity of the ray travelling in the direction $\mu$ and $I_{n}^{-}$represents the specific intensity of the ray travelling in the opposite direction. We select a finite set of values of $\mu\left(\mu_{j}: 1 \leq j \leq m ; 0<\mu_{1}<\mu_{2}<\mu_{3} \cdots \mu_{m}<1\right)$

$$
I_{n}^{+}=\left(\begin{array}{c}
I_{n}\left(\mu_{1}\right) \\
I_{n}\left(\mu_{2}\right) \\
\vdots \\
I_{n}\left(\mu_{m}\right)
\end{array}\right)
$$

and

$$
I_{n}^{-}=\left(\begin{array}{c}
I_{n}\left(-\mu_{1}\right) \\
I_{n}\left(-\mu_{2}\right) \\
\vdots \\
I_{n}\left(-\mu_{m}\right)
\end{array}\right)
$$

are m-dimensional vectors on Euclidean space.

The incident intensity vectors are $I_{n}^{+}$and $I_{n+1}^{-}$. The emergent intensity vectors are $I_{n}^{-}$and $I_{n+1}^{+}$. The emergent radiation field will have the contributions from the internal sources, say, $\Sigma^{+}(n+1, n)$ and $\Sigma^{-}(n, n+1)$ corresponding to the output intensity vectors $I_{n+1}^{+}$and $I_{n}^{-}$respectively.

We assume certain linear operators which reflect and transmit the incident radiation e.g., $t(n, n+1), t(n+1, n), r(n, n+1)$ and $r(n+1, n)$. These operators are calculated based on the physics of the medium. Then we can write the output intensities in terms of the transmitted and reflected input intensities together with the internal sources as

$$
\begin{gathered}
I_{n+1}^{+}=t(n+1, n) I_{n}^{+}+r(n, n+1) I_{n+1}^{-}+\Sigma^{+}(n+1, n) \\
I_{n}^{-}=r(n+1, n) I_{n}^{+}+t(n, n+1) I_{n+1}^{-}+\Sigma^{-}(n, n+1) .
\end{gathered}
$$

The relationship given by equation (24) and equation (25) is called the Interaction Principle. Equation (24) and equation (25) can be written concisely as

$$
\left(\begin{array}{c}
I_{n+1}^{+} \\
I_{n}^{-}
\end{array}\right)=\mathbf{S}(n, n+1)\left(\begin{array}{c}
I_{n}^{+} \\
I_{n+1}^{-}
\end{array}\right)+\Sigma(n, n+1)
$$


where

$$
\mathbf{S}(n, n+1)=\left(\begin{array}{ll}
t(n+1, n) & r(n, n+1) \\
r(n+1, n) & t(n, n+1)
\end{array}\right)
$$

If there is another shell with boundaries $(n+1, n+2)$ adjacent to $(\mathrm{n}, \mathrm{n}+1)$, interaction principle for this shell can be written as (Grant \& Hunt 1969)

$$
\left(\begin{array}{c}
I_{n+2}^{+} \\
I_{n+1}^{-}
\end{array}\right)=\mathbf{S}(n+1, n+2)\left(\begin{array}{c}
I_{n+1}^{+} \\
I_{n+2}^{-}
\end{array}\right)+\Sigma(n+1, n+2)
$$

where $\mathbf{S}(n+1, n+2)$ is similarly defined as in equation (27). If we combine the two shells $(n, n+1)$ and $(n+1, n+2)$ then the interaction principle for the combined shell is written as (for the thickness is arbitrarily defined):

$$
\left(\begin{array}{c}
I_{n+2}^{+} \\
I_{n}^{-}
\end{array}\right)=\mathbf{S}(n, n+2)\left(\begin{array}{c}
I_{n}^{+} \\
I_{n+2}^{-}
\end{array}\right)+\Sigma(n, n+2) .
$$

$\mathbf{S}(n, n+2)$ is called the star product of the two S-matrices $\mathbf{S}(n, n+1)$ and $\mathbf{S}(n+1, n+2)$; and $\mathbf{S}(n, n+2)$ can be written as

$$
\mathbf{S}(n, n+2)=\mathbf{S}(n, n+1) \star \mathbf{S}(n+1, n+2) .
$$

Equation (29) is obtained by eliminating $I_{n+1}^{+}$and $I_{n+1}^{-}$from equation (26) and equation (28). We can write $r$ and $t$ operators for the composite cell as

$$
\begin{aligned}
& t(n+2, n)=t(n+2, n+1)[I-r(n+2, n+1) r(n, n+1)]^{-1} t(n+1, n), \\
& t(n, n+2)=t(n, n+1)[I-r(n, n+1) r(n+2, n+1)]^{-1} t(n+1, n+2), \\
& r(n+2, n)=r(n+1, n)+t(n, n+1)[I-r(n+2, n+1) r(n, n+1)]^{-1} \times \\
& r(n+2, n+1) \text {, } \\
& r(n, n+2)=r(n+1, n+2)+t(n+2, n+1)[I-r(n, n+1) r(n+2, n+1)]^{-1} \times \\
& r(n, n+1)
\end{aligned}
$$

and

$$
\Sigma(n, n+2)=\Lambda(n, n+1 ; n+2) \Sigma(n, n+1)+\Lambda^{\prime}(n ; n+1, n+2) \Sigma(n+1, n+2)
$$

where $I$ is the identity matrix and

$$
\Lambda(n, n+1 ; n+2)=\left(\begin{array}{cc}
t(n+2, n+1)[I-r(n+2, n+1) r(n, n+1)]^{-1} & 0 \\
t(n, n+1) r(n+2, n+1)[I-r(n+2, n+1) r(n, n+1)]^{-1} & I
\end{array}\right)
$$




$$
\Lambda^{\prime}(n ; n+1, n+2)=\left(\begin{array}{cc}
I & t(n+2, n+1) r(n, n+1)[I-r(n, n+1) r(n+2, n+1)]^{-1} \\
0 & r(n, n+1)[I-r(n, n+1) r(n+2, n+1)]^{-1}
\end{array}\right)
$$

and

$$
\Sigma(n, n+1)=\left(\begin{array}{c}
\Sigma^{+}(n+1, n+2) \\
\Sigma^{-}(n, n+1)
\end{array}\right)
$$

Similarly, $\Sigma(n+1, n+2)$ is defined.

In order to obtain physical interpretation of the equations (31) - (34) we expand the operator inverse in a power series. For example,

$$
\begin{gathered}
t(n+2, n)=\sum_{k=0}^{\infty} t_{k}(n+2, n), \\
t_{k}(n+2, n)=t(n+2, n+1)[r(n, n+1) r(n+2, n+1)]^{k} t(n+1, n) .
\end{gathered}
$$

This operator acts on intensities to the right and gives the contribution to $I_{n+2}^{+}$from $I_{n}^{+}$. The term $t_{k}(n+2, n)$ may be recognized as diffuse transmission from $\mathrm{n}$ to $\mathrm{n}+1$, diffuse reflection from the layer $(n, n+1)$, $\mathrm{k}$ times in succession and finally diffuse transmission through $(n+1, n+2)$. Thus $t(n+2, n)$ is the sum of contributions involving scattering of all orders $k=0,1,2, \ldots, \infty$. A similar interpretation can be given for other operators.

If we write $\mathbf{S}(\alpha)$ ( $\alpha$ to designate the cell) then

$$
\mathbf{S}(\alpha \star \beta)=\mathbf{S}(\alpha) \star \mathbf{S}(\beta)
$$

where $\alpha \star \beta$ denotes the region obtained by putting the two cells $\alpha$ and $\beta$ together. If the cells are homogeneous and plane parallel then

$$
\alpha \star \beta=\beta \star \alpha .
$$

In general, star multiplication is non-commutative. However, star multiplication is associative. If we have to add several layers $\alpha, \beta, \gamma, \ldots$ then,

$$
[(\alpha \star(\beta \star \gamma) \star \ldots)]=\mathbf{S}[(\alpha \star \beta) \star \gamma \star \ldots] .
$$

If the medium is optically very thick then we can use what is known as 'doubling method'. For example,

$$
\mathbf{S}\left(2^{P} d\right)=\mathbf{S}\left(2^{P-1} d\right) \star \mathbf{S}\left(2^{P-1} d\right),(P=1,2,3, \ldots)
$$

which means that we can generate the S-matrix for a layer of thickness $2^{P} d$ in $\mathrm{P}$ cycles starting with $\mathbf{S}(d)$ rather than in $2^{P}$ cycles of adding the $\mathbf{S}(d) s$ one by one. For example if $P=10$, then only a fraction $10 / 2^{10} \simeq 10^{-2}$ of the computational work is needed to add $2^{10}$ layers of thickness $\mathrm{d}$.

We expect the reflection and the transmission operators to be non- negative on the physical grounds that intensities are always non-negative. This condition will be satisfied only when the 
optical thickness of the shell is less than certain value called the 'critical size' or $\tau_{\text {crit }}$. If the optical thickness $\tau$ of the shell in question is larger than $\tau_{\text {crit }}$ then we can divide the shell into several sub-shells whose thickness $\tau$ is less than $\tau_{\text {crit }}$ and then use star algorithm to calculate combined response from the sub-shells whose total thickness is T. If, for example, we need the radiation field at internal points in the atmosphere, we shall have to divide the entire medium into as many shells as we need and calculate the radiation field at the $\mathrm{N}$ points in the medium. One can write down the interaction principle for each shell and solve the whole system of equations.

The solution $I_{n+1}^{+}$and $I_{N}^{-}$(for any shell between shell 1 (at the outermost region) and shell N (at the innermost region) are obtained from the relations

$$
I_{n+1}^{+}=r(1, n+1) I_{n+1}^{-}+V_{n+1 / 2}^{+}
$$

and

$$
I_{n}^{-}=t(n, n+1) I_{n+1}^{-}+V_{n+1 / 2}^{-}
$$

with the boundary conditions $I_{N+1}^{-}=I^{-}(a)$. The quantities $r(1, n+1), V_{n+1}^{+}$and $V_{n+1}^{-}$are calculated by employing the initial conditions $r(1,1)=0$ and $V_{1 / 2}^{+}=I^{+}(b)$. The computation is done by the following recursive relations :

$$
\begin{aligned}
r(1, n+1) & =r(n, n+1)+t(n+1, n) r(1, n)[I-r(n+1, n) r(1, n)]^{-1} t(n, n+1), \\
& V_{n+1 / 2}^{+}=t(n+1, n) V_{n-1 / 2}^{+}+\Sigma^{+}(n+1, n)+R_{n+1 / 2} \Sigma^{-}(n, n+1), \\
& V_{n+1 / 2}^{-}=r(n+1, n) V_{n-1 / 2}^{+}+T_{n+1 / 2} \Sigma^{-}(n, n+1),
\end{aligned}
$$

where

$$
\begin{array}{r}
t(n+1, n)=t(n+1, n)[I-r(1, n) r(n+1, n)]^{-1}, \\
r(n+1, n)=r(n+1, n)[I-r(1, n) r(n+1, n)]^{-1}, \\
R_{n+1 / 2}=t(n+1, n) r(1, n), \\
T_{n+1 / 2}=[I-r(n+1, n) r(1, n)]^{-1}, \\
t(n, n+1)=T_{n+1 / 2} t(n, n+1) .
\end{array}
$$

To calculate the radiation field at the internal points we proceed as follows :

(1) Divide the medium into a number of shells (say N) with $\mathrm{N}+1$ boundaries as mentioned earlier. (2) Start calculating the two pairs of reflection and transmission operators $r(n+1, n), r(n, n+1)$, $t(n+1, n)$ and $t(n, n+1)$ in each shell. If the optical thickness of any shell is larger than $\tau_{c r i t}$ then apply star algorithm to use doubling procedure if the medium is homogeneous.

(3) With the boundary condition that $r(1,1)=0$ and $V_{1 / 2}^{+}=I^{+}(b)$ and the $r$ and $t$ operators mentioned in (2) compute recursively $r(1, n+1), V_{1 / 2}^{+}$and $t(n, n+1)$ given in equation (41) to equation (43) from shell 1 to shell $\mathrm{N}$ i.e., from $\mathrm{b}$ to a. 
(4) Next sweep back from a to b calculating the radiation field given in equation (40) with the boundary condition $I_{n+1}^{-}=I^{-}(a)$.

We use a eight point Gaussian quadrature formula for the numerical integration over the angular points and hence $\mu$ and $\mu^{\prime}$ are the zeros of the Legendre polynomials of degree 8 in the interval -1 to +1 . The phase matrix is normalized to 1 .

\section{ROTATION-INDUCED OBLATENESS AND DISK INTEGRATED POLARIZATION}

Once $I_{l}(\mu)$ and $I_{r}(\mu)$ are calculated in any local points, the polarization integrated over the surface of the object can be calculated which should be the observable quantity for point source of light. However, the net polarization would cancel out to zero because of symmetry if the the apparent disk of the object is perfectly spherical. Rapid rotation of the object makes it non-spherical and hence non-zero polarization arises when integrated over the surface.

The oblateness of a rotating object has been discussed by Chandrasekhar (1933) in the context of polytropic gas configuration under hydrostatic equilibrium. For a slow rotator, the relationship for the oblateness $f$ of a stable polytropic gas configuration under hydrostatic equilibrium is given by

$$
f=1-\frac{R_{p}}{R_{e}}=\frac{2}{3} C \frac{\Omega^{2} R_{e}^{3}}{G M}
$$

where $M$ is the total mass, $R_{e}$ is the equatorial radius, $R_{p}$ is the polar radius and $\Omega$ is the angular velocity of the object. $C$ is a constant whose value depends on the polytropic index. For the polytropic index $n=0$, the density is uniform and $C=1.875$. This configuration is known as the Maclaurin spheroid. For a polytropic index of $n=1.0, C=1.1399$, which is appropriate for Jupiter (Hubbard 1984). For non-relativistic completely degenerate gas, $n=1.5$ and $C=0.9669$. Comparisons with detailed structure models (D. Saumon, private communication) show that brown dwarf interiors can be adequately approximated by polytropes with $1<n<1.3$ with the larger $n$ being appropriate for higher gravities. For $\mathrm{n}=1$ and $R_{e}=R_{\text {jup }}$ the oblateness varies from 0.001 to 0.086 when the projected rotational velocity varies from $\mathrm{V}=10$ to $90 \mathrm{kms}^{-1}$ with the surface gravity $g=10^{5} \mathrm{cms}^{-2}$ and it varies from 0.00035 to 0.0287 with $g=3 \times 10^{5} \mathrm{cms}^{-2}$. Similarly, for

$\mathrm{n}=1.5$ and $R_{e}=R_{\text {jup }}$, the oblateness varies from 0.0009 to 0.073 with $g=10^{5} \mathrm{cms}^{-2}$ and it varies from 0.0003 to 0.024 with $g=3 \times 10^{5} \mathrm{cms}^{-2}$ for the same variation in the rotational velocity. It should be noted in this context that at 1 bar pressure level, the oblateness $f$ of Jupiter, Saturn and Earth are 0.065, 0.098 and 0.003 respectively. Barnes \& Fortney (2003) used Darwin-Radau relationship and estimated the oblateness of the exoplanet HD209458b to be about 0.00285 whereas the polytropic approximation with $\mathrm{n}=1$ yields a value of 0.00296 .

Therefore, in the present work, we employ the polytropic approximation of Chandrasekhar with the polytropic index $n=1$, to find out the rotation-induced oblateness because this is appropriate 
for low mass dwarfs and provides an upper limit to the oblateness and hence an upper limit on the degree of polarization for a given rotational velocity. As stated before, the surface gravity of all the models is fixed at $g=10^{5} \mathrm{cms}^{-2}$.

We assume an axisymmetric shape of the object so that it has a rotational invariance around some axis. The radius of any point on the surface is given by

$$
R(\Omega, \mu)=\frac{R_{e}}{\left[1+\left(A^{2}-1\right) \mu^{2}\right]^{1 / 2}}
$$

where $\mathrm{A}$ is the ratio of equatorial radius to the polar radius, i.e., $A=1 /(1-f)$. Using the spherical harmonic expansion around $\theta$ and $\phi$ (Jackson 1975), we find the total flux integrated over the surface as

$$
F_{I}=\frac{1}{2} \sum_{l=0}^{\infty}(2 l+1) P_{l}(\cos i) \int_{-1}^{1} \frac{P_{l}(\mu) d \mu}{\left[1+\left(A^{2}-1\right) \mu^{2}\right]^{1 / 2}} \int_{-1}^{1}\left[I_{l}(\mu)+I_{r}(\mu)\right] \mu P_{l}(\mu) d \mu
$$

and the polarized flux as

$$
F_{Q}=2 \pi \sum_{l=2}^{\infty} \alpha^{2}(l, 2) P_{l}^{2}(\cos i) \int_{-1}^{1} \frac{P_{l}(\mu) d \mu}{\left[1+\left(A^{2}-1\right) \mu^{2}\right]^{1 / 2}} \int_{-1}^{1}\left[I_{l}(\mu)-I_{r}(\mu)\right] \mu P_{l}^{2}(\mu) d \mu
$$

where

$$
\alpha(l, m)=\left[\frac{(2 l+1)(l-m) !}{4 \pi(l+m) !}\right]^{1 / 2},
$$

$P_{l}(\mu)$ is the Legendre polynomial and $P_{l}^{m}(\mu)$ is the associated Legendre polynomial. The disk integrated linear polarization is $p=F_{Q} / F_{I}$.

The disk integrated polarization of a star is investigated by Harrington \& Collins II (1968) who assumed a Roche model wherein the entire gravitational mass is considered to be at the center of the star. The formalism of Harrington \& Collins II (1968) although is valid for stars, is unlikely to be applicable for brown dwarfs and giant gaseous planets because of their very different mass distributions. Nevertheless, we use the formalism of Harrington \& Collins II (1968) and calculate the disk integrated polarization in order to obtain an order of magnitude check in the results with spherical harmonic expansion method. However, the shape function $x(\Omega, \theta)$ in Harrington \& Collins II (1968) is replaced by

$$
x(\Omega, \mu)=\frac{1}{\left[1+\left(1 / A^{2}-1\right) \mu^{2}\right]^{1 / 2}}
$$

so that the shape of the object takes the form of an ellipsoid, as before, and the formalism becomes comparable with the spherical harmonic expansion method. In both the methods we consider $R_{e}=R_{\text {jup }}$ and the mass $\mathrm{M}$ is derived from the surface gravity which is fixed at $10^{5} \mathrm{cms}^{-2}$. 


\section{RESULTS AND DISCUSSION}

The effective temperature of $\mathrm{T}$ dwarfs varies from about $550 \mathrm{~K}$ to $1200 \mathrm{~K}$. Above $1200 \mathrm{~K}$, condensates of various species appears in the photosphere and transition from $\mathrm{L}$ dwarfs to $\mathrm{T}$ dwarfs takes place at about $1300 \mathrm{~K}$ to $1400 \mathrm{~K}$. On the other hand, T dwarf with effective temperature less than $800 \mathrm{~K}$ are likely too faint for image polarimetry. Therefore, in the present work we have considered an effective temperature ranging from $800 \mathrm{~K}$ to $1200 \mathrm{~K}$. However, for a comparison with L dwarfs of effective temperature $1400 \mathrm{~K}$ which have condensates in their photosphere and hence should show detectable amount of polarization in the optical because of dust scattering, we have included the case for $T_{\text {eff }}=1400 \mathrm{~K}$ without condensates. This case provides a point of comparison to illustrate how polarization differs with or without dust scattering. The surface gravity of typical field brown dwarfs varies from $10^{5}$ to $3 \times 10^{5} \mathrm{cms}^{-2}$ (e.g., Saumon \& Marley (2008)). The departure from rotation-induced sphericity decreases with the increase in surface gravity (Sengupta \& Kwok 2005). In order to maximize the amount of polarization and hence to obtain an upper limit on the degree of polarization of $\mathrm{T}$ dwarfs, we have fixed the surface gravity at $\mathrm{g}=10^{5} \mathrm{cms}^{-2}$.

The numerical method used to calculate the local polarization provides unconditional stability for plane-parallel stratification of the atmosphere subject to the condition that each shell has less than the critical optical depth. This is achieved by dividing the shells with high optical depth into sub-shells with optical depth less than the critical optical depth. Usually, $\tau<0.1$ serves the purpose. A convenient test of the efficacy of the numerical method is to study the case of conservative scattering. In a purely scattering medium, the physical system must neither create nor destroy energy. For this purpose we apply unpolarized incident radiation at the boundary of the inner radius of the shell and no radiation is incident at the outer shell. For a conservatively scattering medium, the total flux that is introduced at the inner boundary is found to be equal within nine-th decimal figure to the sum of the flux that comes out of the outer boundary and the backscattered flux at the inner boundary. The polarization is zero when the phase function $\mathbf{P}\left(\mu, \mu^{\prime}\right)=\mathbf{1}$ i.e., when isotropic scattering is considered.

Finally, the total emergent flux given by

$$
F=F_{l}+F_{r}=\int_{-1}^{1}\left[I_{l}(\mu)+I_{r}(\mu)\right] \mu d \mu^{\prime}
$$

matches exactly with the emergent flux obtained by Marley (2000) and Stephens et al. (2009) for scalar radiative transfer case. In figure 1, we present the emergent flux for condensate-free atmospheres with effective temperature ranging from $800 \mathrm{~K}$ to $1400 \mathrm{~K}$. As it is well known, the emergent flux in the optical falls rapidly with the decrease in wavelength owing to the presence of alkaline elements (Burrows, Marley \& Sharp 2000; Liebert et al. 2000). Therefore, T dwarfs are extremely faint at shorter wavelengths.

Figure 2(a-d) shows the polarization profiles at different directions for different effective temperatures. The polarization is maximum when $\theta$, the angle between the direction of the radiation field and the symmetry axis is $90^{\circ}$. However, the degree of polarization falls steeply with the 
decrease in $\theta$. At $\theta=90^{\circ}$, significant polarization is found even up to a wavelength of $1.5 \mu \mathrm{m}$ for the coolest $\mathrm{T}$ dwarf $\left(T_{\text {eff }}=800 \mathrm{~K}\right)$. But at a slightly smaller value of $\theta$, polarization becomes insignificant at wavelength longer than even $0.6 \mu \mathrm{m}$. The angular dependency of the polarization for different effective temperature is presented in figure 3. As the angle between the direction of the radiation field and the symmetry axis decreases, the anisotropy in the radiation field reduces drastically because of the fact that at smaller angles, mostly the unscattered photons emerged out and that the probability of scattering increases as $\theta$ increases. The probability of scattering is maximum at $\theta=90^{\circ}$.

Figure 2(a-d) also shows that the degree of polarization decreases as the effective temperature of the object increases. In fact, this is true, not only at the outermost boundary but also at any depth inside the atmosphere. In figure 4 we present the atmospheric depth dependence of the polarization at a particular wavelength $(\lambda=0.6 \mu \mathrm{m})$ and at a direction $\mu=0.02$. The degree of polarization for $T_{\text {eff }}=800 \mathrm{~K}$ remains almost constant up to 1 bar of pressure level and then falls rapidly to zero. As the effective temperature increases, the degree of polarization falls rapidly to zero at a higher altitude or at a lower pressure level. The physical explanation of this feature becomes clear from figure 5 wherein the variation of the single scattering albedo $\omega_{0}$ with respect to the atmospheric pressure $\mathrm{P}$ and temperature $\mathrm{T}$ is presented. For an object with $T_{\text {eff }}=1200 \mathrm{~K}$, the scattering albedo is almost constant and its value is about one above a pressure level of $10^{-1}$ bar. Bellow this depth, it asymptotically falls to zero. $\omega_{0}$ becomes zero at deeper region as one goes from hotter to cooler objects. This means, the scattering albedo remains non zero up to much deeper region as the objects become cooler and hence contribution to the polarization originates from deeper region in cooler objects. As a consequence, the degree of polarization is higher in cooler objects as shown in figure 4 .

The polarization profile discussed above is calculated at a local point of the surface of the atmosphere stratified into plane-parallel geometry. This can be observable only if the object can be spatially resolved. However, a distant stellar object cannot be spatially resolved and it appears to be a point source of light to an earth based observer. Therefore only the disk averaged polarization is the observable quantity. However, if the apparent disk of a stellar object is perfectly spherical, because of symmetry, the net polarization averaged over the spherical disk would cancelled out.

High resolution spectroscopic analysis of T dwarfs by Zapatero Osorio et al. (2006); del Burgo et al. (2009) shows that just like L dwarfs, these objects are also fast rotators. As a consequence, net non-zero polarization should arise when integrated over the apparent disk because rotation induces distortion in the stellar disk. In figure 6 and figure 7, we present the disk integrated polarization of $\mathrm{T}$ dwarfs at an edge on view, i.e., at an inclination of $90^{\circ}$ with rotational velocity $\mathrm{V}=90$ and 60 $\mathrm{kms}^{-1}$ respectively. The degree of polarization is maximum when the inclination angle is $90^{\circ}$, i.e., at an edge on view to an observer and it decreases with the decrease in the inclination angle as can be seen from figure 8. Harrington \& Collins II (1968) also reported the same feature. Figure 6 and Figure 7 shows that the degree of polarization of $\mathrm{T}$ dwarf with any spectral type is non-zero only at wavelengths shorter than $0.6 \mu \mathrm{m}$. As the rotational velocity increases, the oblateness of 
an object with the same mass, radius and polytropic index increases. As a consequence with increasing rotational velocity there is less cancellation of polarization over the surface, yielding higher polarization. At the same time, the effect of limb darkening and gravity darkening too increases and consequently the degree of polarization increases further. However, because of the fact that $\mathrm{T}$ dwarfs are extremely faint at wavelengths shorter than $0.6 \mu \mathrm{m}$, it would be extremely difficult to detect such a low polarization of $\mathrm{T}$ dwarfs, especially if the inclination angle is off the edge on view.

The synthetic spectra calculated by using one dimensional plane- parallel atmospheric stratification match well with the observed spectra. However, a three dimensional approach is worth investigating in order to achieve better understanding of the effect of limb darkenning and the polarization integrated over the disk of a non-spherical object.

\section{CONCLUSIONS}

In the present paper, we have discussed the results of our investigation on multiple scattering polarization of cloudless $\mathrm{T}$ dwarfs. We predict that in the absence of condensates in the photosphere of T dwarfs later than about type T3 (Stephens et al. 2009), non-zero polarization would arise only at wavelengths shorter than $0.6 \mu \mathrm{m}$ - a spectral region where these dwarfs are exceptionally dark. In order to calculate the maximum possible polarization of $\mathrm{T}$ dwarfs, we have considered a polytropic equation of state with index $n=1$ which is the minimum permissible value of $n$ for representing the matter distribution in brown dwarfs. Consequently, the rotation-induced oblateness is maximized for a given rotational velocity.

We find that even with a rotational velocity as high as $90 \mathrm{kms}^{-1}$, the maximum amount of disk integrated polarization that arises at an inclination angle of $90^{\circ}$, is of the order $10^{-4}$, too small to be detected in the B-band. The degree of polarization would be greatest for the coolest objects and it would decrease with increasing effective temperature. The results support the claim by Sengupta (2003); Sengupta \& Kwok (2005) that the polarization detected in the optical region (R- and I-band) of $\mathrm{L}$ dwarfs has negligible contribution from atomic and molecular scattering. Therefore, if linear polarization in R- or I-band of $\mathrm{T}$ dwarfs is detected in future, it will imply the presence of condensates in the photosphere of $\mathrm{T}$ dwarfs. Although a magnetic field in principle might produce polarization, $\mathrm{T}$ dwarf atmospheres are highly neutral (Gelino et al. 2002) and thus the presence of magnetic field would not give rise to detectable amount of polarization in the optical (Menard et al. 2002). Finally, polarization could play a crucial role in measuring the loss of condensates from brown dwarf atmospheres as we expect L- and early T-type dwarfs to show linear polarization in R- and I-band by dust scattering in the phototosphere whereas mid-type and later $\mathrm{T}$ dwarfs would not show any polarization at wavelength greater than $0.6 \mu \mathrm{m}$. The detailed investigation of the polarization of $\mathrm{L}$ dwarfs by multiple dust scattering will be presented in a forthcoming paper. 


\section{Acknowledgements}

S.S. is thankful to A. Peraiah for useful discussions. S.S. also acknowledges support by TIARA/ASIAA-National Tsing-Hua University, Taiwan where a part of this work was done. M.M.

acknowledges support from the NASA Planetary Atmospheres Program. Thanks are due to the referee for constructive comments.

\section{REFERENCES}

Ackerman \& Marley 2001, ApJ, 556, 872.

Allard et al. 2001, ApJ, 556, 357.

Bailer-Jones, C. A. L. 2004, A \& A, 419, 703.

Barnes, J. W. \& Fortney, J. J. 2003, ApJ, 588, 545.

Basri, G. et al. 2000, ApJ, 538, 363.

Burrows, A. \& Sharp, C. 1999, ApJ, 512, 843.

Burrows, A., Marley, M. \& Sharp, C. 2000, ApJ, 531, 438.

Burrows, A., Hubbard, W. B., Lunine, J. I., \& Liebert, J. 2001, Rev. Mod. Physics, 73, 719.

Chabrier, G., \& Baraffe, I. 2000, ARA\&A, 38, 337.

Chandrasekhar, S. Radiative Transfer (New York: Dover, 1960).

Chandrasekhar, S. 1933, MNRAS, 93, 539.

Cushing, M. C. et al. 2008, ApJ, 678, 1372.

del Burgo, C. et al. 2009, arXiv:0903.4461.

Freedman, R. S., Marley, M. S., \& Lodders, K. 2008, ApJS, 174, 504.

Gelino, C. R., Marley, M. S., Holtzman, J. A., Ackerman, A. S., \& Lodders, K. 2002, ApJ, 577, 433.

Goldman, B. et al. 2009, A \& A, 502, 929.

Grant, I. P. \& Hunt, G. E. 1969, Proc. Roy. Soc. Lond. A313, 183.

Grant, I. P. \& Hunt, G. E. 1969a, Proc. Roy. Soc. Lond. A313, 199.

Grant, I. P. \& Peraiah, A. 1972, MNRAS 160, 239. 
Harrington, J. P., \& Collins II, G. W. 1968, ApJ, 151, 1051.

Hubbard, W. B. 1984, Planetary Interiors (New York;Van Nostrand Reinhold)

Jackson, J. D., Classical Electrodynamics 2nd edn. (New York: Wiley, 1975).

Kirkpatrick, J. D. et al.1999, ApJ 519 , 834.

Kirkpatrick, J. D. 2005, ARA\&A, 43, 195.

Liebert, J., 2000, ApJ, 533, L155.

Lodders, K. 1999, ApJ, 519, 793.

Lunine, J. I., Hubbard, W. B. \& Marley, M. S. 1986, ApJ, 310, 238.

Marley, M. S., et al. 1996, Science, 272, 1919.

Marley, M. S. 2000, in ASP Conf. Ser. 212, From Giant Planets to Cool Stars, ed. C. A. Griffith \& M. S. Marley (San Francisco : ASP),152

Marley, M. S. \& Leggett, S. K. 2008, Arxiv e-print, arXiv:0803.1476.

Marley, M. S. et al. 2002, ApJ, 568, 335.

Menard et al. 2002, A \& A, 396, L35.

Mohanty, S. \& Basri, G. 2003, ApJ, 583, 451.

Peraiah, A., \& Grant, I. P. 1973, J. Inst. Maths. Appl. 12, 75.

Preisendorfer, R. W., Radiative transfer on discrete spaces (Pergamon, Oxford 1965)

Redheffer, R. M., J. 1962, Math. Phys. 41, 1.

Reiners, A. \& Basri, G. 2008, ApJ 684, 1390.

Sengupta, S. 2003, ApJ, 585, L155.

Sengupta, S. \& Krishan, V. 2001, ApJ, 561, L123.

Sengupta, S. \& Kwok, S. 2005, ApJ, 625, 996.

Saumon, D. \& Marley, M. S. 2008, ApJ, 689, 1327.

Stephens, D. C. et al. 2009, ApJ, 702, 154.

Tata, R. et al. 2009, A \& A, (in press).

Tsuji, T., Ohnaka, K., Aoki, W., \& Nakajima, T. 1996, A\& A, 308, L29 
Tsuji, T. 2002, ApJ, 575, 264.

Zapatero Osorio et al. 2005, ApJ, 621, 445.

Zapatero Osorio et al. 2006, ApJ, 647, 1405.

This preprint was prepared with the AAS $\mathrm{LAT}_{\mathrm{E}} \mathrm{X}$ macros v5.2. 


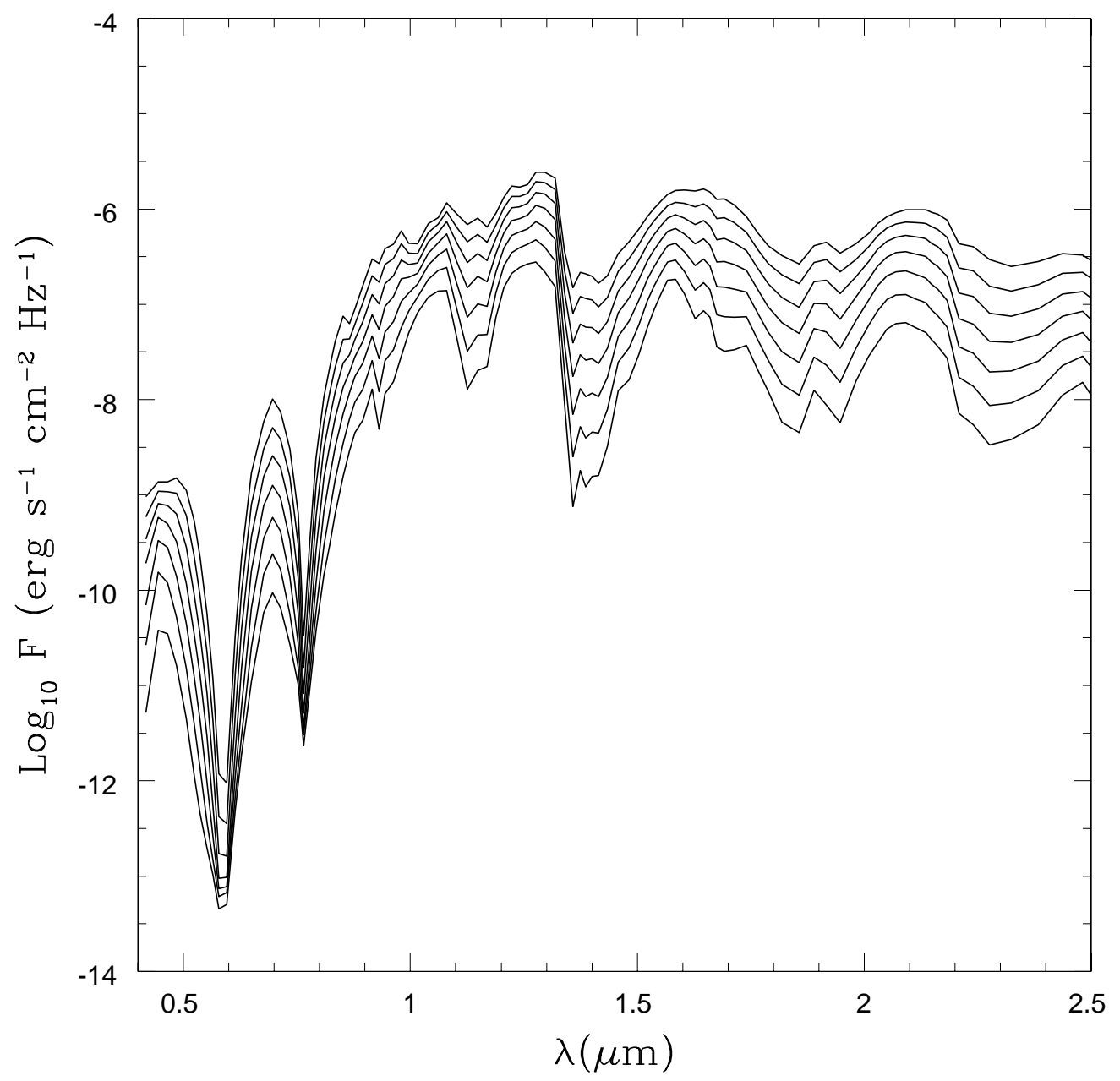

Fig. 1. - The emergent flux as a function of wavelength for $\mathrm{T}$ dwarfs with different effective temperature. From top to bottom, the solid lines represent the flux for $T_{\text {eff }}=1400,1300,1200,1100,1000,900$ and $800 \mathrm{~K}$ respectively. 


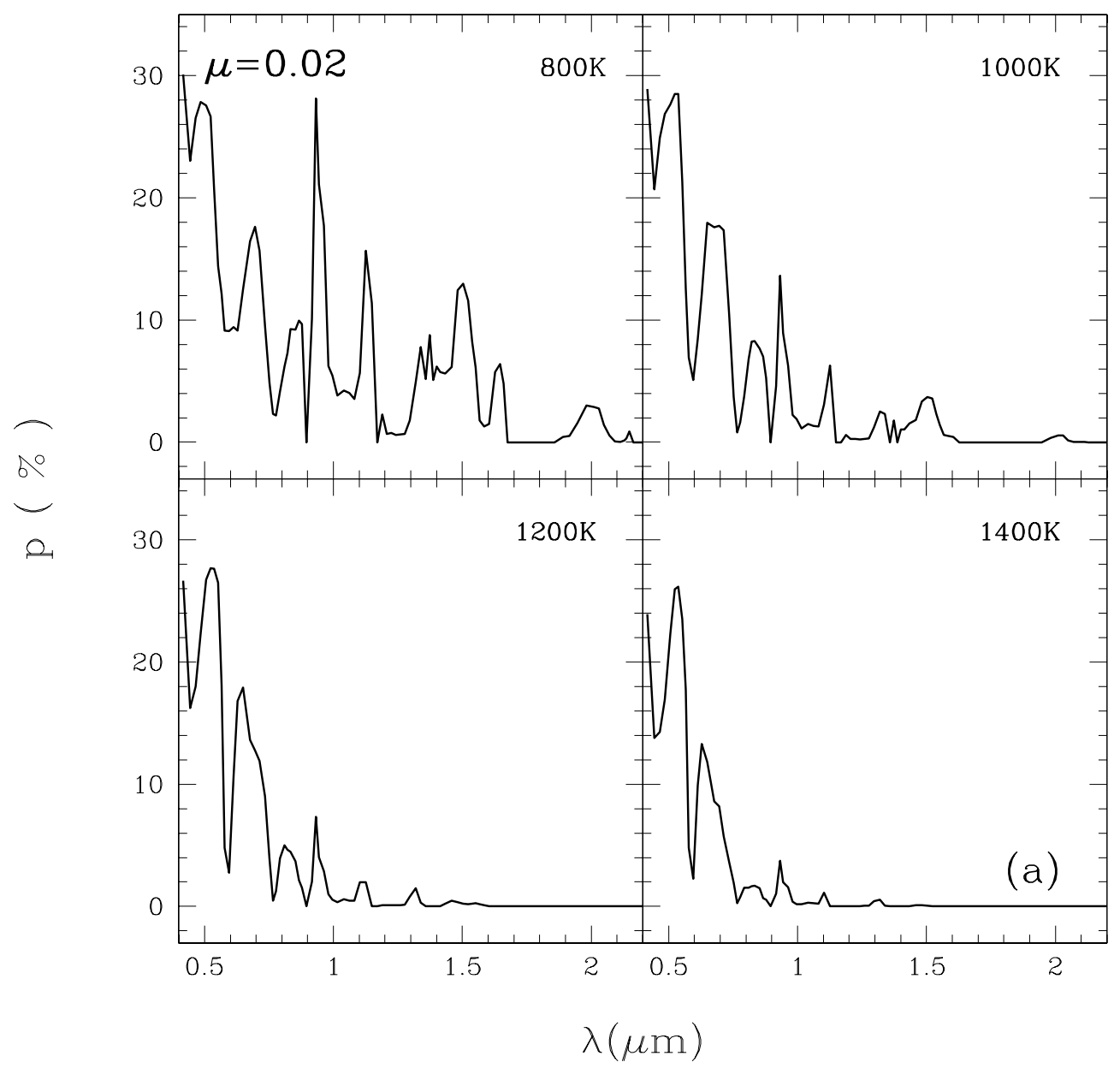

Fig. 2a.- Percentage degree of polarization along $\mu=0.02$ for $\mathrm{T}$ dwarfs with effective temperature $800 \mathrm{~K}, 1000 \mathrm{~K}, 1200 \mathrm{~K}$ and $1400 \mathrm{~K}$. 


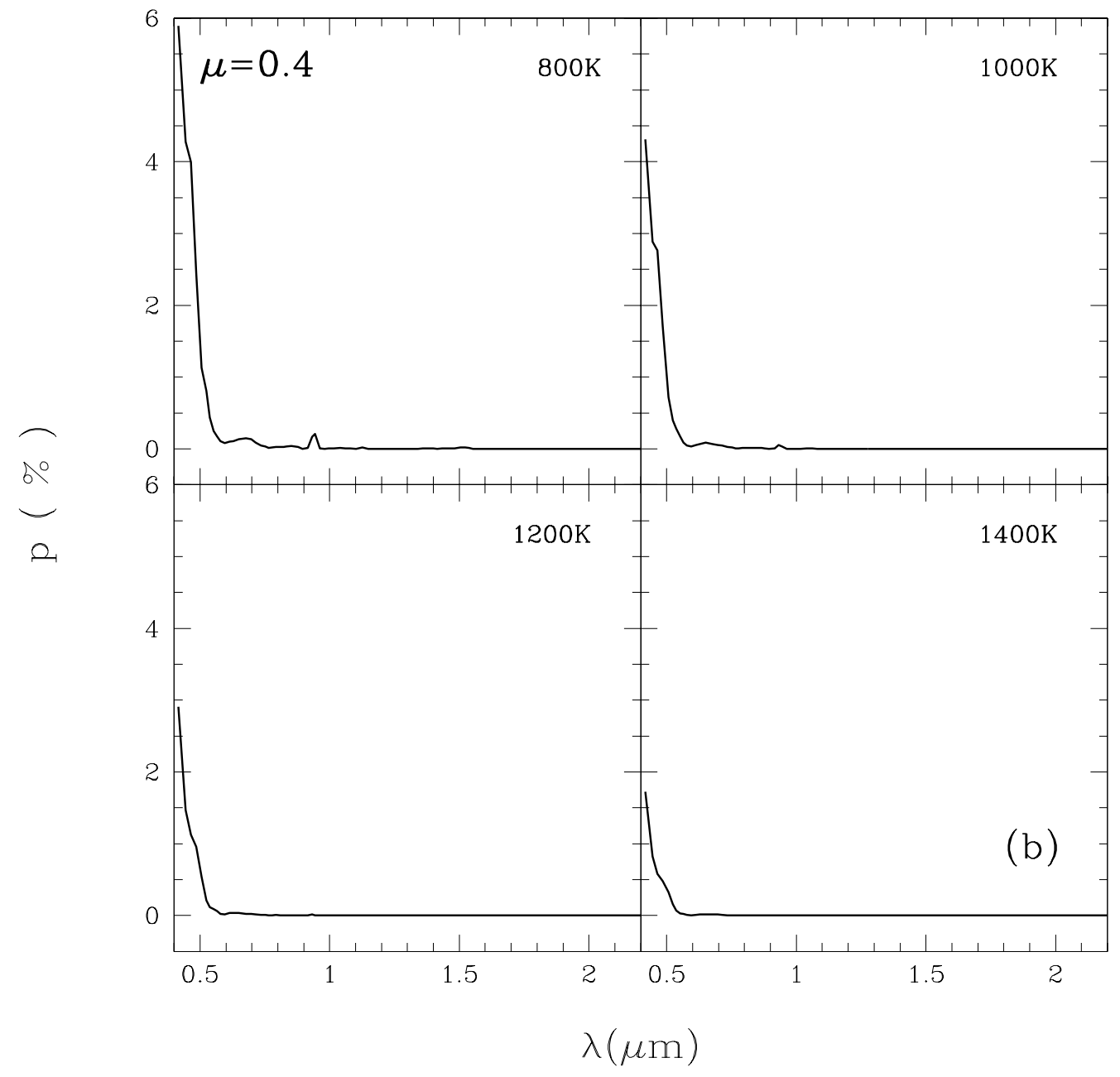

Fig. 2b.- Same as figure 2a but along $\mu=0.4$. 


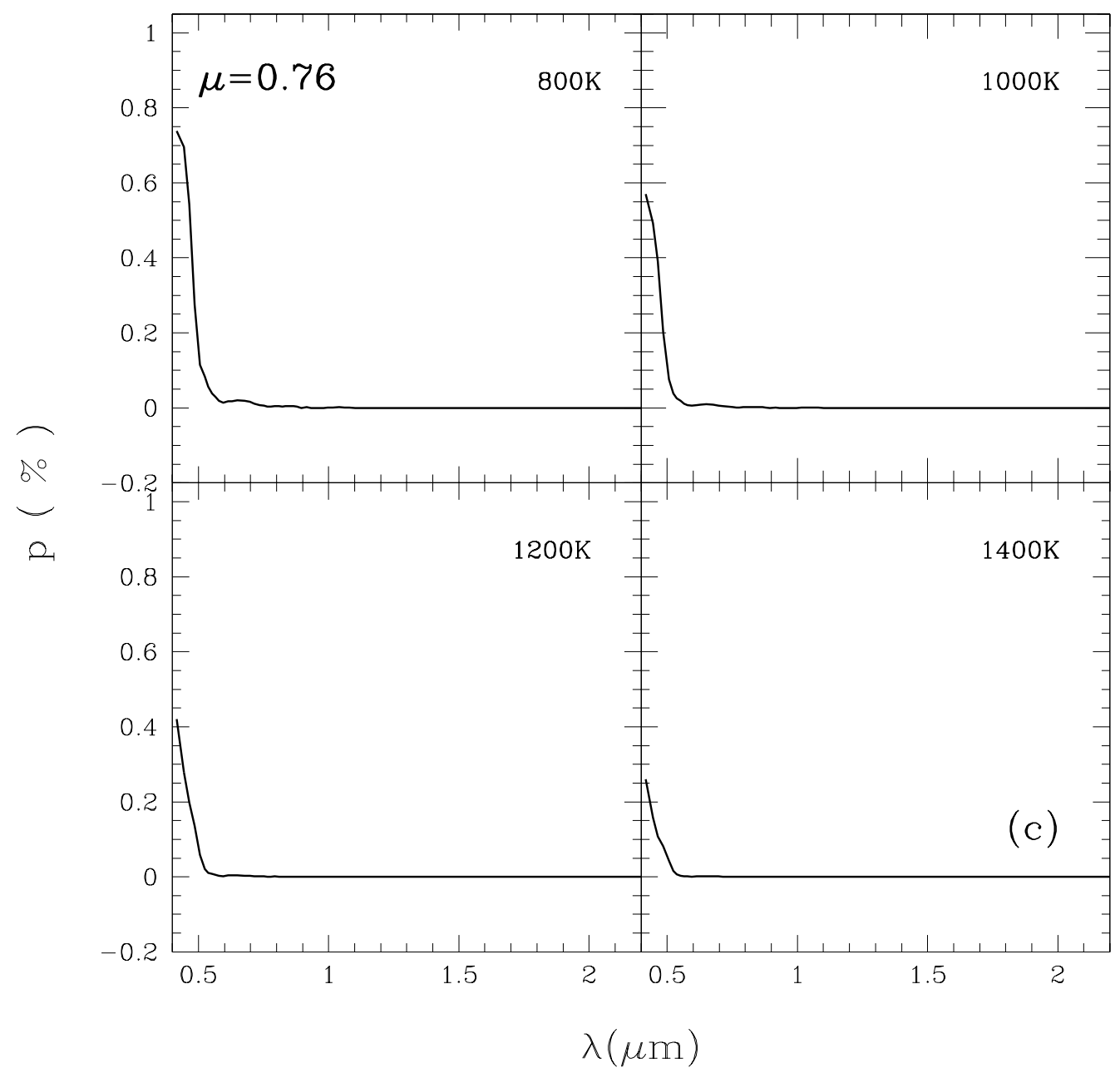

Fig. 2c. - Same as figure 2a but along $\mu=0.76$. 


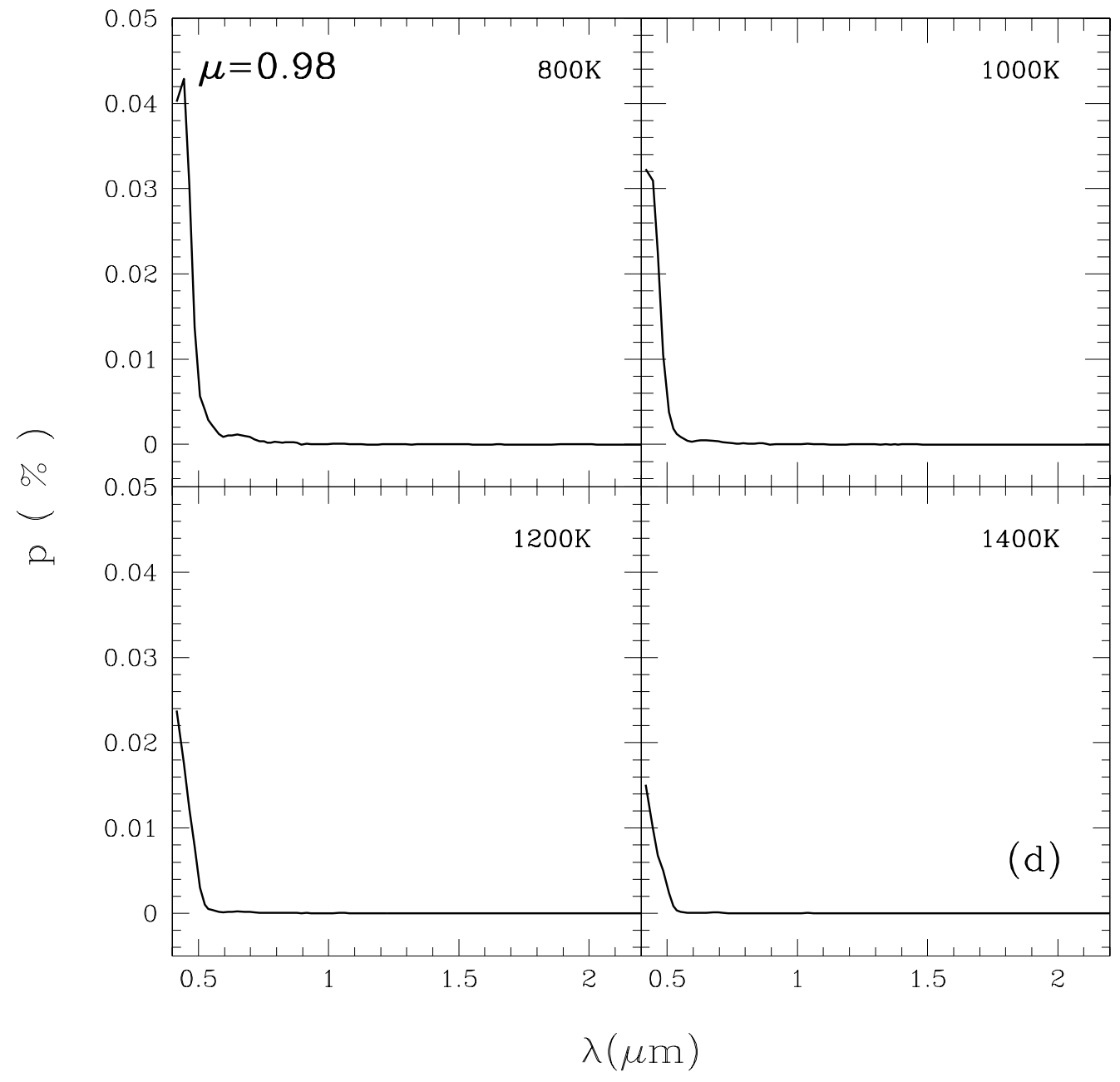

Fig. 2d.- Same as figure 2a but along $\mu=0.98$. 


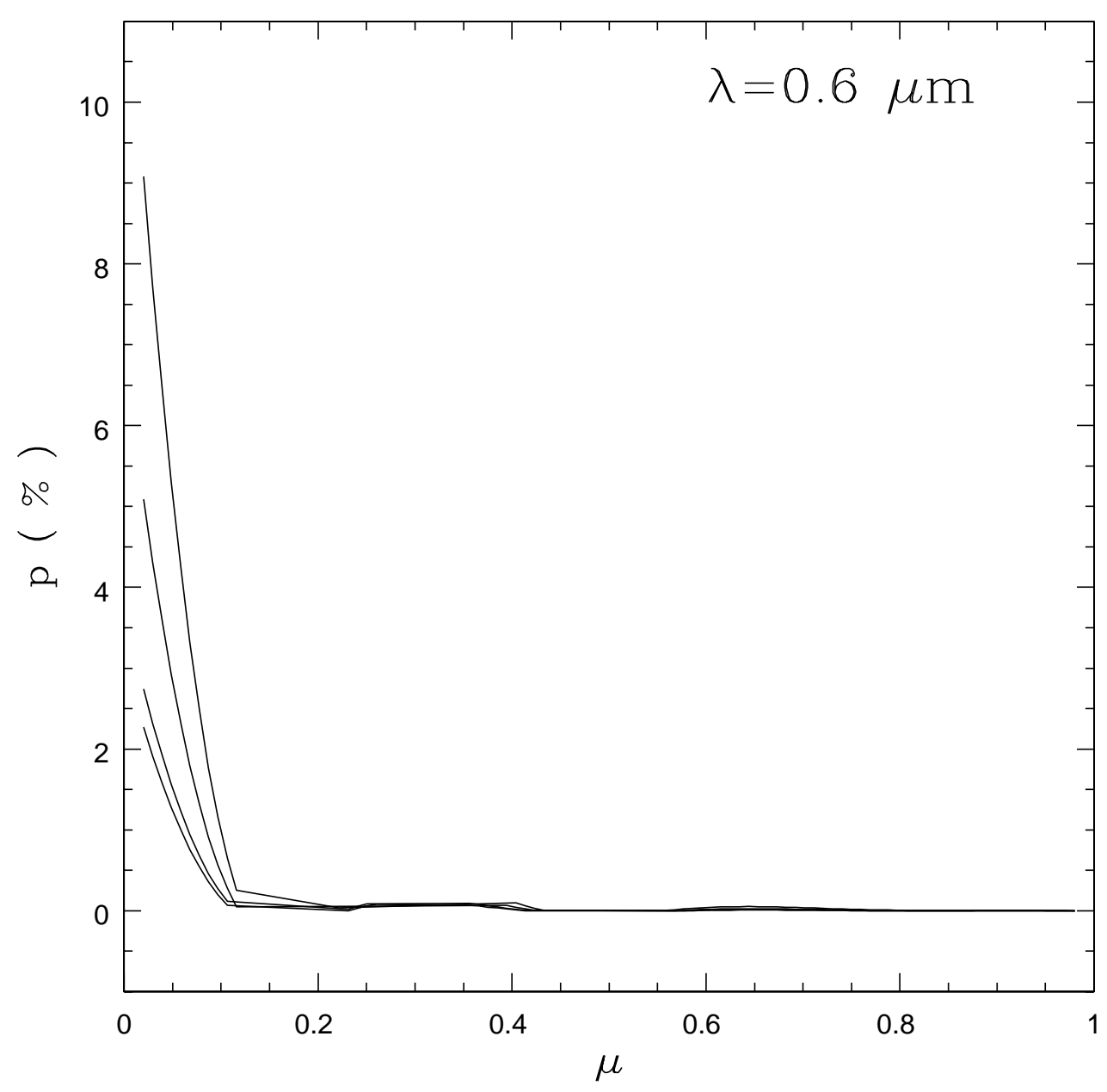

Fig. 3.- Percentage degree of polarization at different $\mu=\cos \theta$ where $\theta$ is the angle between the direction of the radiation and the symmetry axis. The polarization is calculated at a wavelength $\lambda=0.6 \mu \mathrm{m}$. 


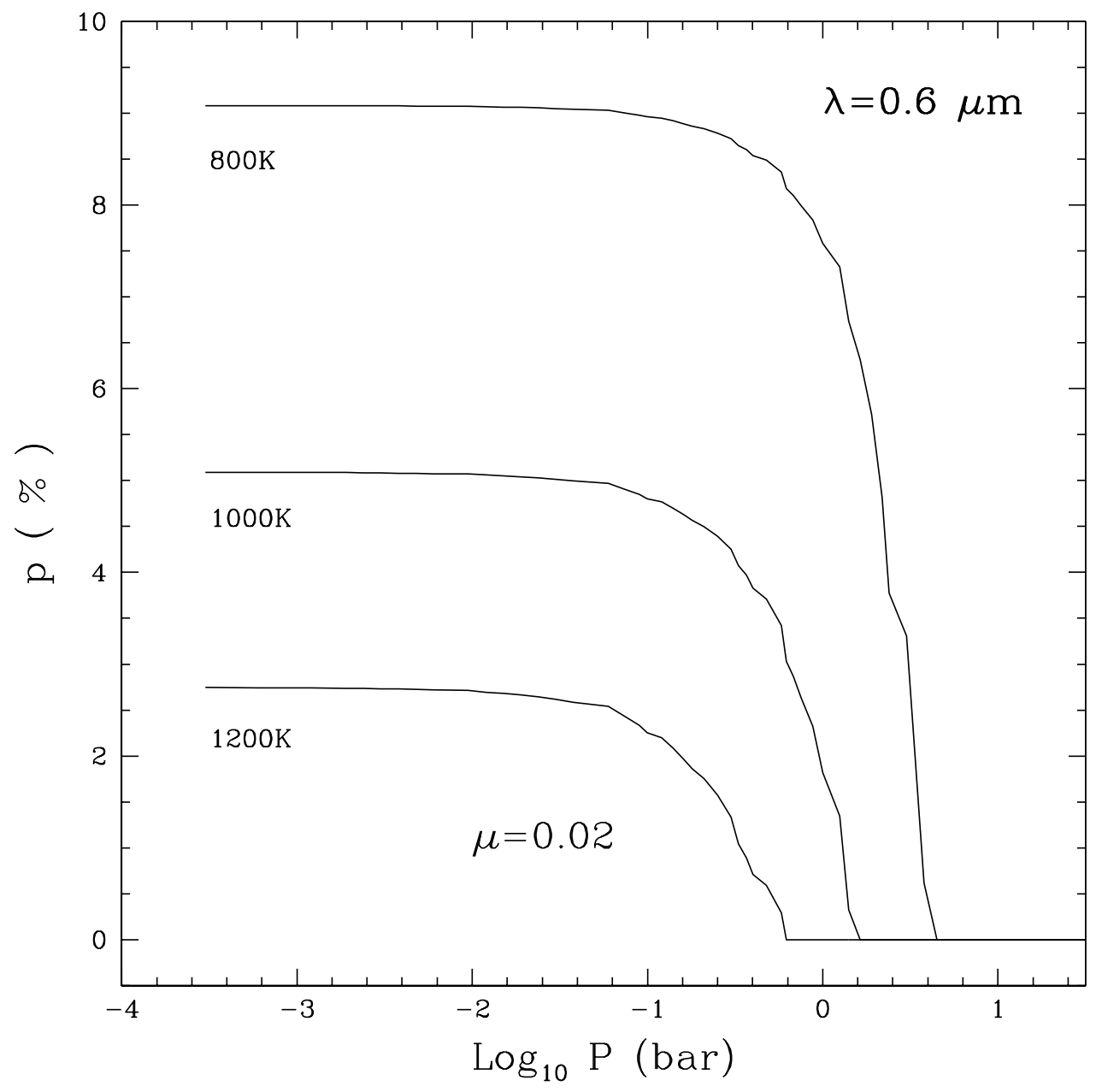

Fig. 4. - Percentage degree of polarization along $\mu=0.02$ at different atmospheric pressure level. The polarization is calculated at a wavelength $\lambda=0.6 \mu \mathrm{m}$. 


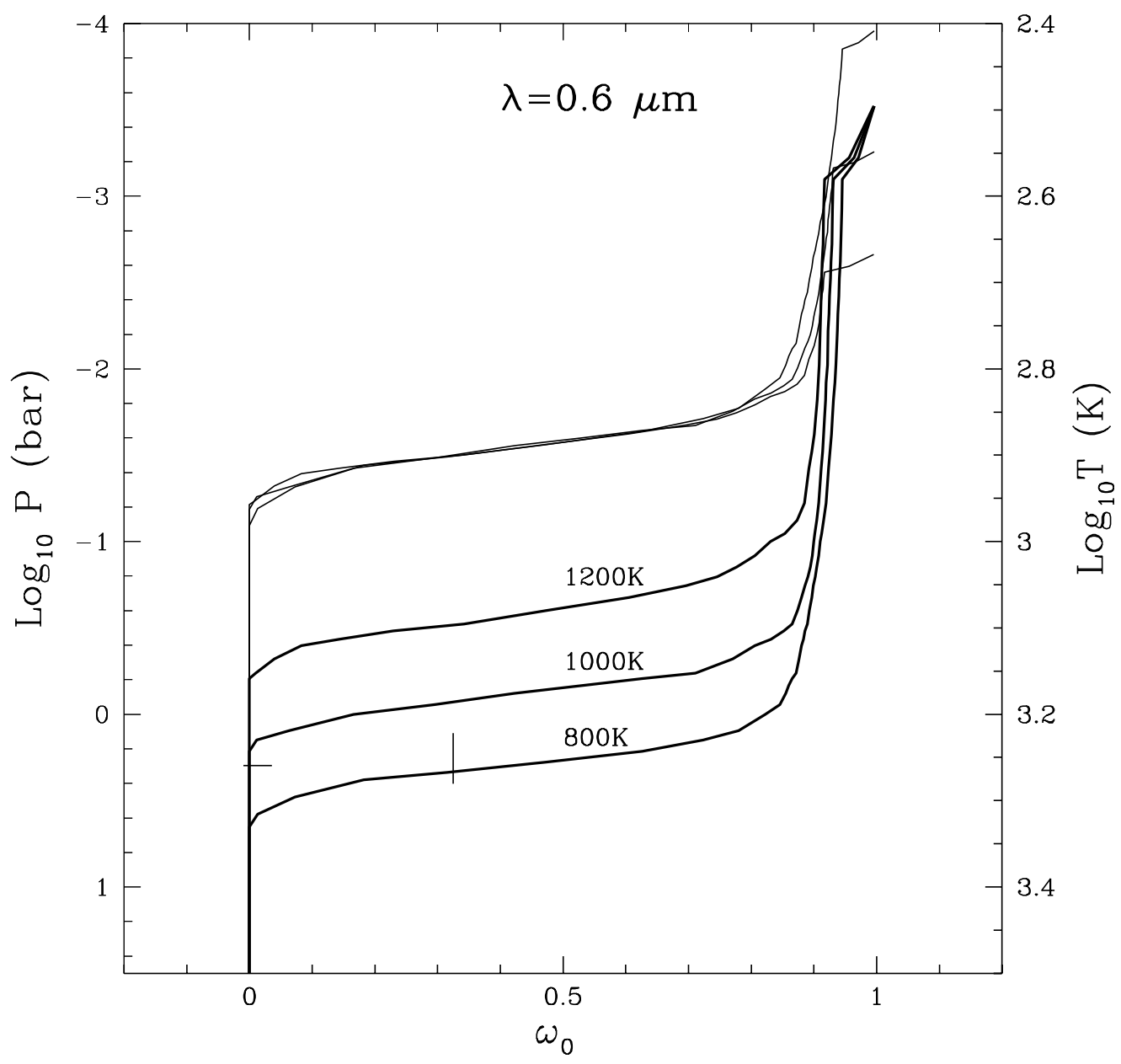

Fig. 5.- Atmospheric depth dependence of single scattering albedo $\omega_{0}$ at a wavelength $\lambda=0.6$ $\mu \mathrm{m}$. The thick solid lines represent $\omega_{0}$ vs. atmospheric pressure $\mathrm{P}$ scaled in the left hand side axis. The dashes over the lines indicate the location of the photosphere. The thin solid lines represent the variation of $\omega_{0}$ with respect to the atmospheric temperature $\mathrm{T}$ scaled in the right hand side axis. 


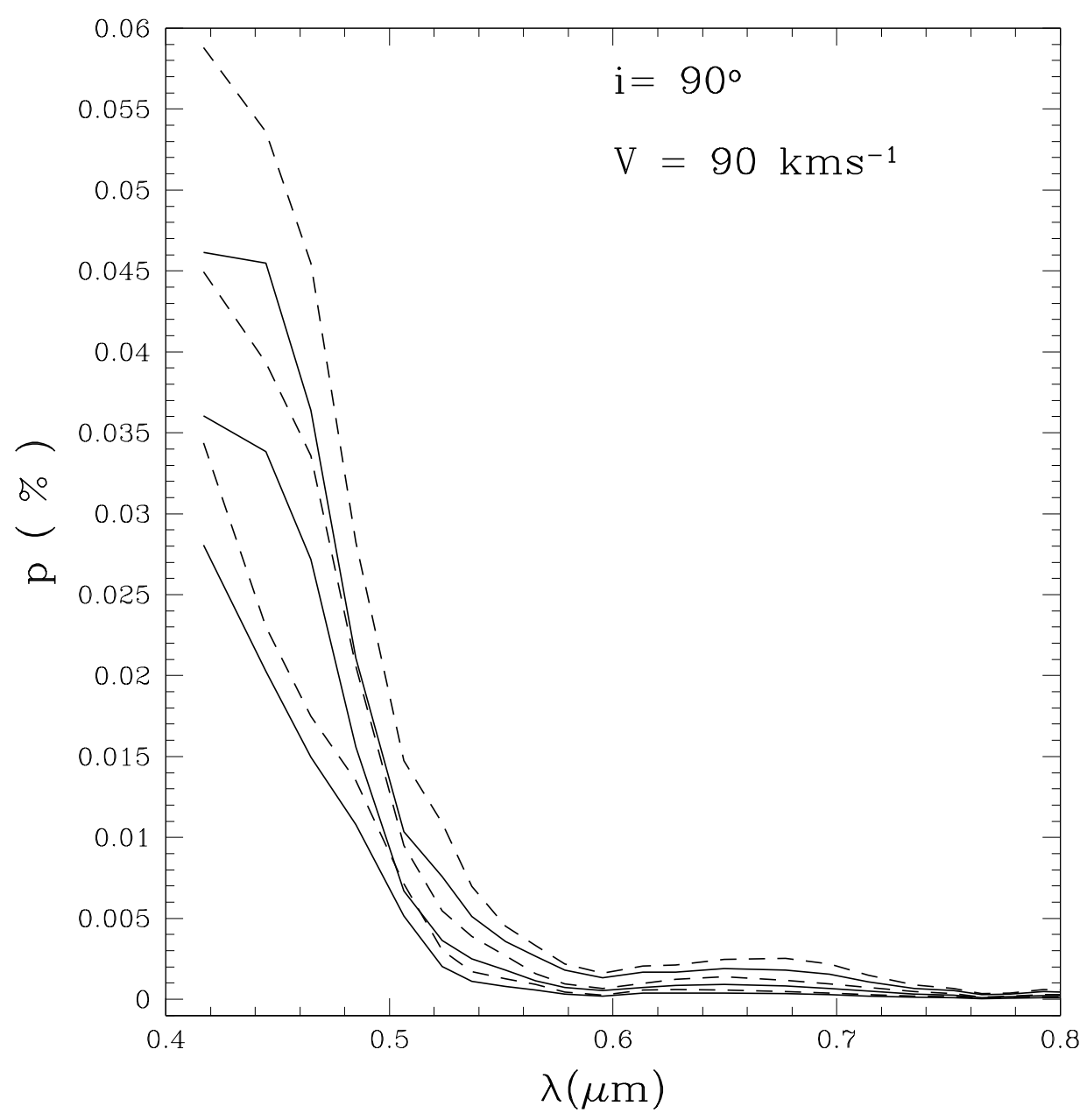

Fig. 6. - The disk integrated polarization at an inclination angle $i=90^{\circ}$ and for a rotational velocity $V=90 \mathrm{kms}^{-1}$. The solid lines represent the percentage polarization calculated by using the spherical harmonic expansion method while the broken lines represent that calculated by using the modified Harrington-Collins method. From top to bottom the lines represent the disk integrated polarization of $\mathrm{T}$ dwarfs with effective temperature $800 \mathrm{~K}, 1000 \mathrm{~K}$ and $1200 \mathrm{~K}$ respectively. 


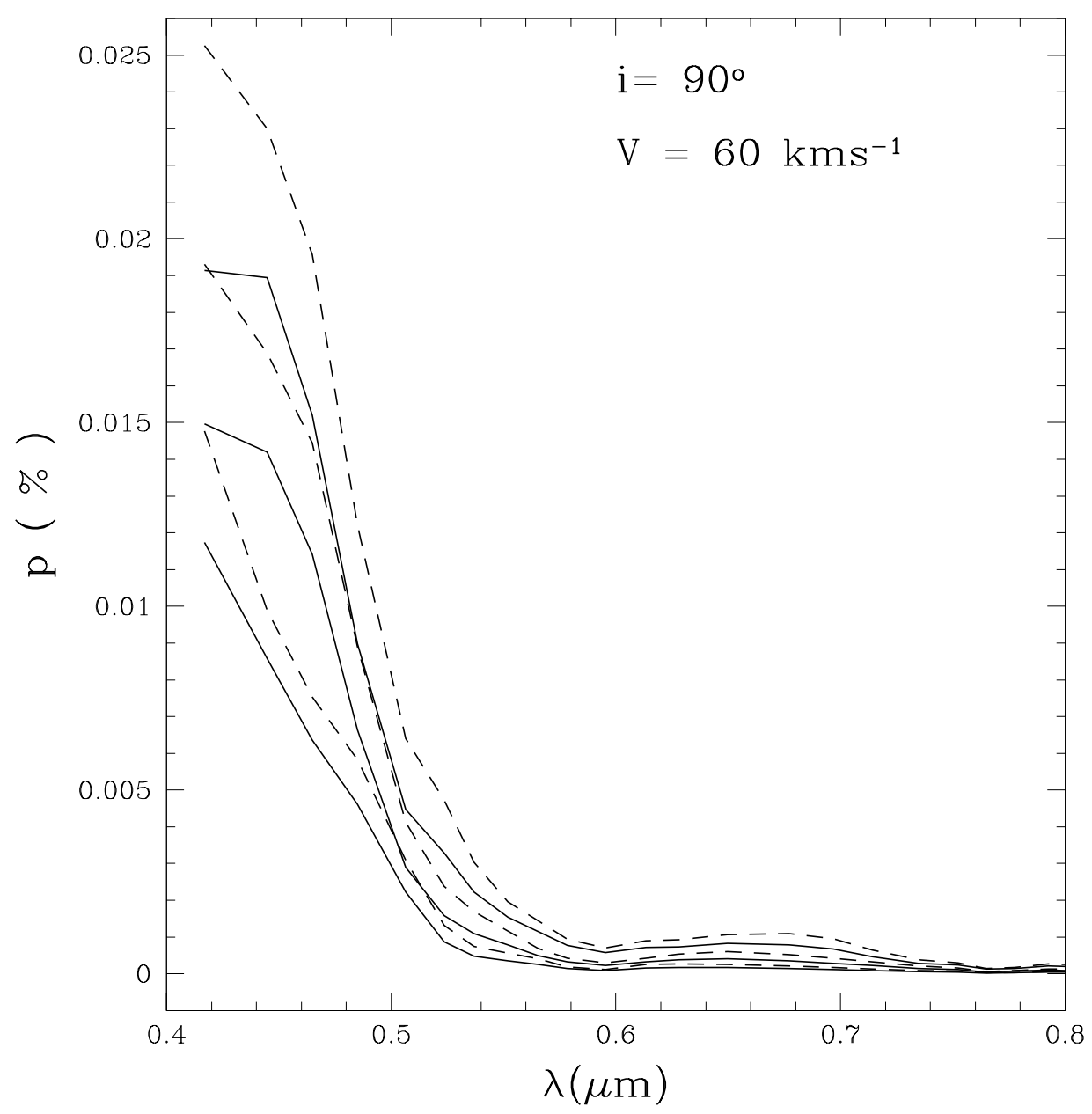

Fig. 7.- Same as figure 6 but with $V=60 \mathrm{kms}^{-1}$. 


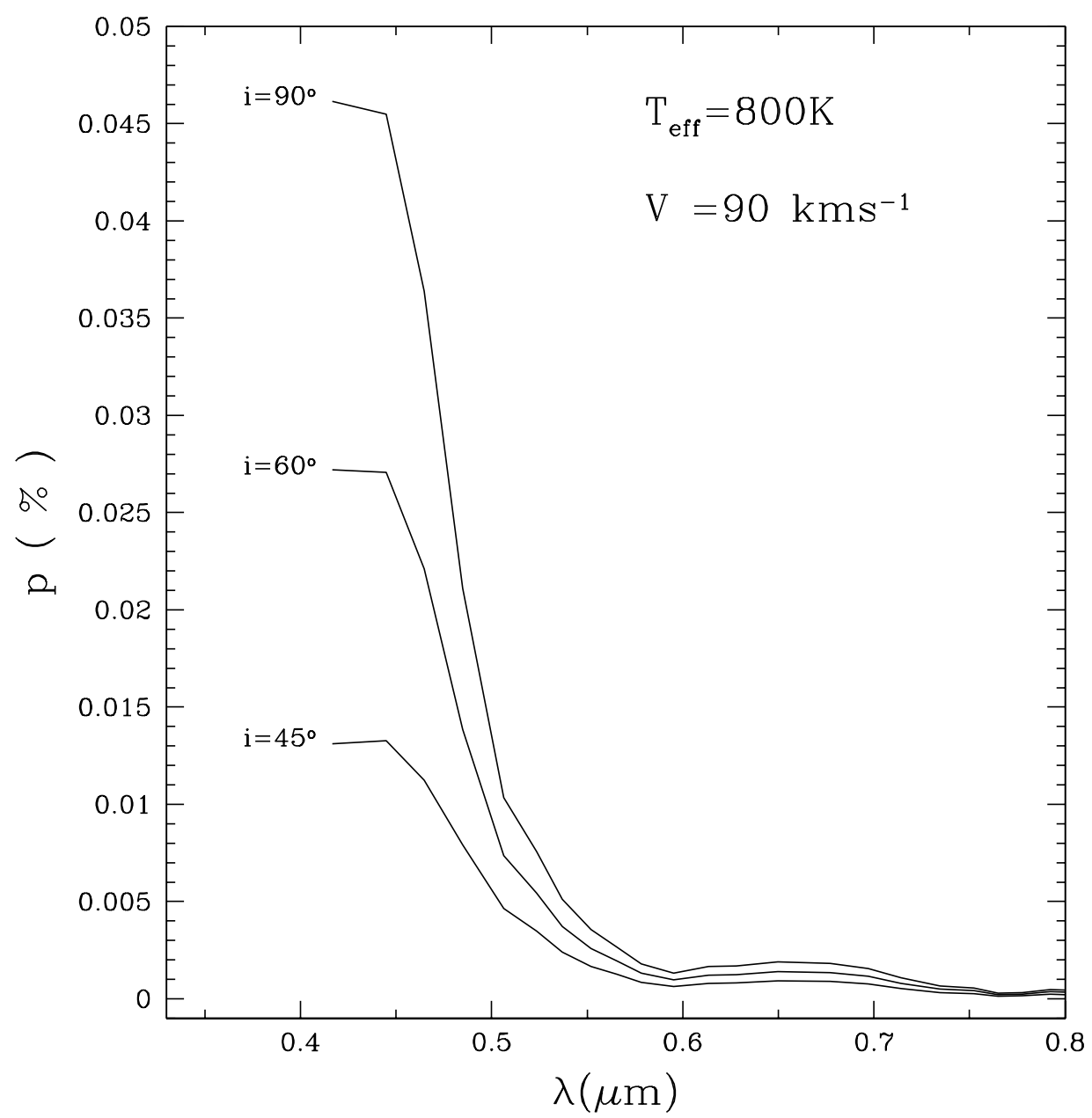

Fig. 8.- Disk integrated polarization at different inclination angles i. The polarization profiles shown here are calculated by using the spherical harmonic expansion method. 